\title{
Tamoxifen monitoring studies in breast cancer patients by micellar
}

\section{liquid chromatography}

\author{
Josep Esteve-Romero ${ }^{*}$, Enrique Ochoa-Aranda ${ }^{2}$, Devasish Bose ${ }^{3}$, Maria Rambla-Alegre', \\ Juan Peris-Vicente ${ }^{1}$, Adrià Martinavarro-Domínguez² \\ ${ }^{1}$ Química Bioanalítica, QFA, ESTCE, Campus Riu Sec, Universitat Jaume I, 12071, Castelló, Spain \\ ${ }^{2}$ Hospital Provincial, Dr. Clara, 12003, Castelló, Spain \\ ${ }^{3}$ Criminology and Forensic Science, Dr. H.S. Gour University, Sagar, India
}

Keywords: Micellar liquid chromatography, Pharmaceuticals, Plasma, Tamoxifen, UV.

* Corresponding author. Tel.: +34-964728093; Fax: +34-964728066

E-mail address: josep.esteve@gfa.uji.es 


\begin{abstract}
A simple micellar liquid chromatographic procedure is described to determine tamoxifen in plasma. To perform the analysis, tamoxifen solutions were diluted in water and UVirradiated for $20 \mathrm{~min}$ to form the photocycled derivative with a phenanthrene core which shows intense fluorescence. Samples were then directly injected, thus avoiding long extraction and experimental procedures. The resolution from the matrix was performed with a mobile phase containing $0.15 \mathrm{M} \mathrm{SDS}-7 \%$ n-butanol at $\mathrm{pH} 3$ running at $1.5 \mathrm{ml} / \mathrm{min}$ through a $\mathrm{C} 18$ column at $40^{\circ} \mathrm{C}$. Detection was carried out by fluorescence, and the excitation and emission wavelengths were 260 and $380 \mathrm{~nm}$, respectively. The chromatographic analysis time was less than 15 min. The analytical methodology was validated following the International Conference on Harmonisation of Technical Requirements for Registration of Pharmaceuticals for Human Use (ICH) guidelines. The response of the drug in plasma was linear and in the $0.5-15 \mu \mathrm{g} / \mathrm{ml}$ range, with $r^{2}>0.999$. Accuracy and precision were $<9 \%$ in both cases. The limits of detection and quantification $(\mathrm{ng} / \mathrm{ml})$ were 50 and 150 in plasma, respectively. The method developed herein shows no interferences by endogenous compounds. Finally, the analytical method was used to determine the amount of tamoxifen in the plasma of several breast cancer patients from a local hospital.
\end{abstract}




\section{Introduction}

Tamoxifen (TAMO; MW = $371 \mathrm{~g} / \mathrm{mol})$, or trans-1-(4- -dimethylaminoethoxyphenyl) -1,2-diphenylbut-1-ene (Fig. 1), has proved most successful in helping women with breast cancer. Tamoxifen is a non-steroidal triphenylethylene antiestrogen that competitively inhibits oestradiol binding to the high affinity oestrogen receptor [1-3]. Chemically, tamoxifen is a white, odourless, fine crystalline powder [4]. It has been approved by the US Food and Drug Administration (FDA) in 1978 as a chemopreventive in women at high risk of breast cancer, and is now prescribed to women at high risk of this disease [1,3,5]. Tamoxifen is supplied in pharmaceuticals as oral tablets of tamoxifen citrate. This compound has a molecular weight of 563.4 and $\mathrm{a} \mathrm{KK}_{\mathrm{a}}=8.85$ and is moderately soluble in water and acid [6].

The long-term safety of TAMO is a subject of controversy given its oestrogen activity which can cause endometrial cancer and thromboembolic diseases [7]. Moreover, it has numerous side effects: mild nausea, vaginal dryness, irritability, headache, fever, inability to concentrate, and fatigue [8]. Moreover, the drug is not effective in some patients who respond to therapy and eventually relapse [9]. TAMO is metabolised by cytochrome P450 enzymes in vivo to several compounds such as N-desmethyltamoxifen (DMT), 4-hydroxy-tamoxifen (OHT) and tamoxifen-N-oxide, $\alpha$-hydroxytamoxifen, $\mathrm{N}$-didesmethyltamoxifen and 4-hydroxy$\mathrm{N}$-desmethyltamoxifen (endoxifen). The activity and side effects caused by ingesting the drug is caused not only by TAMO, but also by its biological active metabolites $[1,3,5,10]$.

Several methods based on HPLC have been developed to determine tamoxifen in pharmaceuticals [6], biological matrixes like cell tissues [11], urine [12,13], serum [5,9,14] plasma [1,2,6,15-17], and even environmental samples [18]. Some pharmacokinetic studies have also been performed [10,16,19]. With biological fluids analyses, these methodologies include the previous removal of the matrix by liquid/liquid with organic solvent $[1,2,6,13,14,16]$, a solid phase $[13,17]$ the extraction of molecular imprinted polymers [12], or the injection of the sample after deproteinisation [5,15]. Tamoxifen detection can be carried out by mass spectrometry [5,14], UV-spectrophotometry [6,12,17] and fluorescence. In this 
case, the sample must be previously on-line [2,15,16] or off-line [1] irradiated by UV at 254 $\mathrm{nm}$ in order to convert tamoxifen into its photocyclation product (with a phenanthene core) [20] which shows intense fluorescence.

Micellar liquid chromatography (MLC) is an alternative to these drug determination methods in pharmaceuticals and physiological fluids. Micelles tend to bind proteins competitively by releasing protein-bound drugs and proteins, rather than precipitating into the column, and being solubilized and washed harmlessly away to elute with the solvent front. This allows the direct injection of the sample, thus avoiding complex and time-consuming extraction steps. Another advantage is that the use of micellar mobile phases is non-toxic, non-flammable, biodegradable and relatively inexpensive if compared to aqueous-organic solvents [21]. Two selected studies show the use of micellar mobile phases with direct injection when applied to the determination of drugs in pharmaceuticals [22], serum [23,24] and plasma [25].

This work aims to develop a fast, easy, accurate and reliable experimental procedure using micellar mobile phases to quantify tamoxifen in the plasma of breast cancer patient samples. The analyte must be separated from other sample compounds in a short time and with high sensitivity. The proposed procedure benefits from the main advantage of it being associated with the use of micellar media, for instance fast and easy preparation, and the use of a smaller amount of pollutant organic modifier. This methodology has been validated following the ICH Guidelines [26] in terms of linearity, limits of detection (LOD) and quantification (LOQ), intra- and inter-day precision, selectivity and recovery.

\section{Experimental}

\subsection{Reagents and solutions}

Tamoxifen was supplied by Sigma (St. Louis, MO, USA). The other reagents used were sodium dodecyl sulphate (SDS) (Merck, Darmstadt, Germany), sodium dihydrogenphosphate monohydrate, $\mathrm{HCl}$ (Panreac, Barcelona, Spain), $\mathrm{NaOH}$ (Scharlab, Barcelona), and methanol (J.T. Baker, Holland). Ultrapure water (Millipore S.A.S., Molsheim, France) was used to prepare the aqueous solutions and mobile phases. 
Tamoxifen standards were prepared by solubilising the appropriate amount in ultrapure water, and the mixture was ultrasonicated for 2 minutes. These solutions were prepared in amber flasks to avoid the extensive photochemical degradation of the drug. Fresh solutions were prepared on a weekly basis.

\subsection{Apparatus}

The chromatograph was an Agilent Technologies Series 1100 (Palo Alto, CA, USA) equipped with a quaternary pump, a degasifier for the mobile phase, an autosampler, and a temperature controller for the column module coupled to a fluorescence detector. A Kromasil $5 \mathrm{C} 18$ column was used of a $5 \mu \mathrm{m}$ particle size and $150 \mathrm{~mm} \times 4.6 \mathrm{~mm}$ i.d. (Scharlab). The chromatographic signals were acquired and processed with the HP ChemStation (Rev. B.03.01).

An ultrasonic bath was used to dissolve the standards (model Ultrasons-H, Selecta, Abrera, Spain) and the $\mathrm{pH}$ of the solutions was measured with a Crison GLP 22 (Barcelona) potentiometer equipped with a combined $\mathrm{Ag} / \mathrm{AgCl} /$ glass electrode. The analytical balance used was a Mettler-Toledo AX105 Delta-Range (Greifensee, Switzerland).

The samples were irradiated with a Phillips original home solaria UV lamp (Philips, Eindhoven, The Netherlands).

\subsection{Chromatographic Conditions}

Tamoxifen was separated and quantified using a mobile phase containing 0.15 M SDS and 7\% (v/v) n-butanol buffered at $\mathrm{pH} 3$ (adjusted using suitable volumes of $0.1 \mathrm{M} \mathrm{HCl}$ ), running at a constant flow of $1.5 \mathrm{ml} / \mathrm{min}$. The mobile phase was filtered through $0.45 \mu \mathrm{m}$ nylon membranes (Micron Separations, Westboro, MA). The Kromasil 5 C18 column head pressure was 150 bars. The column temperature was kept constant at $40 \pm 0.5^{\circ} \mathrm{C}$. The excitation and emission wavelengths were 260 and $380 \mathrm{~nm}$, respectively.

\subsection{Sample collection}


Blood samples were collected with DB SST Tubes (BD Vacutainer Systems, Plymouth, UK) and centrifuged at $3000 \mathrm{rpm}$ for $5 \mathrm{~min}$ at $4^{\circ} \mathrm{C}$; plasma was kept at $4^{\circ} \mathrm{C}$.

\subsection{Experimental procedure}

A $100 \mu \mathrm{L}$ volume of the solution containing the analyte (standard or sample) was placed into a small glass vial insert, pulled point glass with $6 \mathrm{~mm}$ diameter (Agilent), and $100 \mu \mathrm{L}$ of ultrapure water were added. The mixture was irradiated at $254 \mathrm{~nm}$ for $20 \mathrm{mi}$ at $40 \mathrm{~W} \mathrm{n}$. Then the solution was diluted with $0.3 \mathrm{ml}$ of ultrapure water, and $20 \mu 1$ were injected into the chromatographic system.

\section{Results and discussion}

\subsection{Optimising the experimental conditions}

\subsubsection{Irradiation time}

Samples were irradiated to form the corresponding phenanthrene derivative which shows strong fluorescence. However, this product was unstable, thus the irradiation time had to be adjusting by considering the final yield of the reaction and the decomposition of the derivative. Tamoxifen standards were irradiated several times and the area of the corresponding peak was measured. As shown in Figure 2, maximum fluorescence was obtained after $20 \mathrm{~min}$ of irradiation.

\subsubsection{Mobile phase selection}


The mobile phase selection was based on the resolution of compounds and a suitable analysis time.

The octanol-water partition coefficient of tamoxifen is 6.64 [27], which means that it is hydrophobic in nature and whose retention in micellar liquid chromatography is expected to be high. An aqueous 0.15 SDS mobile phase buffered at $\mathrm{pH} 3$ was tested, and tamoxifen was eluted after a long time with poor resolution. Then an alcohol was needed to elute the analyte in an appropriate time with a high resolution. Using n-pentanol, tamoxifen eluted too quickly and overlapped with the protein band. So n-butanol was selected as an organic modifier. Finally, sodium dodecyl sulphate $0.15 \mathrm{M}-7 \% \mathrm{n}$-butanol (v/v) at $\mathrm{pH} 3$ was selected as the optimum mobile phase in which the analyte eluted at approximately 14 min with an adequate shape.

\subsection{Method validation}

Validation studies were performed according to the ICH Tripartite guidelines [26], and included linearity, the limits of detection (LOD) and quantification (LOQ), intra- and inter-day precision, selectivity and recovery.

\subsubsection{Selectivity}

To study the matrix effects in the plasma of the possible co-eluting compounds, ten blanks sample were analysed (Fig. 3a). The protein band appeared before the tamoxifen retention time, and there were no peaks near the tamoxifen retention time. A plasma sample spiked with $10 \mu \mathrm{g} / \mathrm{ml}$ of tamoxifen was analysed. The tamoxifen peak may be observed as being sufficiently separated from the other peaks, thus avoiding overlapping (Fig. 3b).

\subsubsection{Linearity and sensitivity}

For calibration purposes, a blank plasma sample was spiked at 9 concentration levels in the range of $0.5-15 \mu \mathrm{g} / \mathrm{ml}$. The slope and intercept were obtained by a correlation between the areas of the chromatographic peak of tamoxifen of the triplicate injection vs. the concentration 
by least square linear regression. Calibration was repeated five times (preparing the sample on each occasion) over a 2-month period, and each calibration level was analysed six times. LOD and LOQ were set as three and ten times the standard deviation of the blank, respectively. The regression curves and limits were:

$$
A=(1040 \pm 30)[T A M O]-(316 \pm 51) r^{2}=0.9991(\mathrm{LOD}=50 \mu \mathrm{g} / \mathrm{ml} ; \mathrm{LOQ}=150 \mu \mathrm{g} / \mathrm{ml})
$$

where $\mathrm{A}$ is in arbitrary units and the concentration is provided in $\mu \mathrm{g} / \mathrm{ml}$.

\subsubsection{Precision and accuracy}

The intra- and inter-day accuracy and precision of the proposed methodology were determined with plasma samples spiked at three concentration levels $(1 ; 2.5 ; 5 \mu \mathrm{g} / \mathrm{ml})$. The intra-day analysis was determined by injecting aliquots of these samples six times on the same day, while the inter-day analyses correspond to the average of five measurements of the intraday values taken over a 3-month period. The results, expressed as variation coefficients for accuracy and relative error for precision, are shown in Table 1. The data show good accuracy (below 7\%) and adequate precision (2.5-8.5\%) for both matrixes, which prove useful for routine analyses.

\subsection{Applying the methodology to plasma patients}

Plasma studies were performed following an oral administration of a single dose of 10 $\mathrm{mg}$ of the tamoxifen drug to several breast cancer patients. Samples were taken over a 24-hour period. The results are shown in Table 2. Figure $3 \mathrm{~d}$ depicts the chromatogram obtained by analysing samples of a breast cancer patient. Tamoxifen can be clearly quantified without interferences. 


\section{Conclusions}

Micellar liquid chromatography has proved a suitable technique to analyse tamoxifen in human plasma. One advantage of this procedure is the possibility of injecting a diluted sample into the chromatographic system, thus avoiding long, tedious extractions. After sample irradiation to improve sensitivity, the analyte was satisfactorily resolved using a mobile phase of $0.15 \mathrm{M} \mathrm{SDS}-7 \%$ n-butanol at $\mathrm{pH} 3$ from the matrix in an analysis time of under $15 \mathrm{~min}$. Validation was performed according to the ICH guidelines with satisfactory results in terms of linearity, selectivity, precision and accuracy. The limit of detection and the lineal interval range were sufficient to detect the usual amount of tamoxifen in patients' plasma. Moreover, this method is relatively inexpensive and uses a smaller amount of toxic organic modifiers, thus making it more interesting.

\section{Acknowledgements}

This study has been supported by projects CTQ2007-64473/BQU funded by the Spanish Ministry of Education and Science (MEC) and EMR-15(1)/2005/C-06 funded by the Directorate of Forensic Science (MHA, GoI) at the Dr. H.S. Gour University, Sagar, India. Maria Rambla-Alegre also wishes to thank the MEC for the FPU grant. 


\section{References}

1. Zhu YB., Zhang Q., Zou J.J., Yu C.X., Xiao D.W. (2008) J. Pharmaceutical Biomed. Anal. 46: $349-355$

2. Lee K.L., Ward B.A., Desta Z., Flockhart D.A., Jones D.R. (2003) J. Chromatogr. B 791: $2545-253$

3. Jordan V.C. (2007) Steroids 72: 829-842

4. American Hospital Formulary Service (1988), Drug Information, American Society of Health-System Pharmacists, Bethesda, MD

5. Gjerde J., Kisanga E.R., Hauglid M., Holm P.I., Mellgren G., Lien E.A. (2005) J. Chromatogr. A 1082: 6-14

6. De Santana D.P., Carvalho Braga R.M., Strattmman R., Muñiz-Albuquerque M., Galindo Bedor D.C., Bastos Leal L. (2008) Quim. Nova 31: 47-52

7. Cuzik J., Forbes J., Edwards R. (2002) Lancet 360: 817-824

8. Aebi S., Pagani O. (2007) Drugs 67: 1393-1401

9. MacCallum J., Cummings J., Dixon J.M., Miller W.R. (1997) J. Chromatogr. B 698: 269275

10. Desta Z., Ward B.A., Soukhova N.V., Flockhart D.A. (2004) J. Pharmacol. Exp. Ther. 310: $1062-1075$

11. Sharma M. (2000) Biochemical and Biophysical Research Communications 273: 40-44

12. Claude B., Morina P., Bayoudh S., Ceaurriz J. (2008) J. Chromatogr. A 1196-1197: 81-88

13. Li X.F., Carter S., Dovichi N.J., Zhao J.Y., Kovarik P., Sakuma T. (2001) J. Chromatogr. A 914: 5-12

14. Teunissen S.F., Rosing H., Koornstra R.H.T., Linn S.C., Schellens J.H.M., Schinkel A.H., Beijnen J.H. (2009) J. Chromatogr B 877: 2519-2529

15. Fried K.M., Wainer I. W. (1994) J. Chromatogr. B 655: 261-268

16. Nieder M., Jaeger H. (1987) J. Chromatogr. B 413: 207-217

17. Merle O., Faure N., Guitton J., Burke M. D., Ollagnier M. (1998) Analytical Letters 31: 
$2679-2690$

18. Hilton M.J., Thomas K.V. (2003) J. Chromatogr. A 1015: 129-141

19. Perez E.A, Gandara D.R., Edelman M.J., O'Donnell R., Lauder I.J., DeGregorio M. (2003) Cancer Invest. 21: 1-6

20. Salamoun J., Macka M., Nechvátal M., Matousek M., Knesel L. (1990) J. Chromatogr. A 514: $179-187$

21. Esteve Romero J., Carda Broch S., Gil Agustí M., Capella-Peiró M.E., Bose D. (2005) Trends Anal. Chem. 24: 75-91

22. Gil-Agusti, M., Carda-Broch, S., Capella-Peiró, M.E., Esteve-Romero, J. (2006) J. Pharmaceutical Biomed. Anal. 41: 1235-1242

23. Capella-Peiró M.E., Gil-Agustí M., Martinavarro-Dominguez A., Esteve-Romero J. (2002) Anal. Biochem. 309: 261-268

24. Rambla-Alegre M., Gil-Agustí M.T., Capella-Peiró M.E., Carda-Broch S., Esteve-Romero J. (2006) J. Chromatogr. B 839; 89-94

25. Rambla-Alegre M., Esteve-Romero J., Carda-Broch S. (2009) Anal. Chim. Acta 633; 250256

26. ICH validation guideline. http://www.ich.org/cache/compo/276-254-1.html

27. Clarke's Analysis of Drugs and Poisons (2004), Pharmaceutical Press, London UK 


\section{FIGURE CAPTIONS}

Figure 1. Structure of tamoxifen.

Figure 2. First-order plots of the fluorescence of tamoxifen versus the UV irradiation time.

Figure 3. Chromatograms obtained by analysing the tamoxifen samples by the proposed methodology: a) blank plasma sample; b) plasma spiked with $10 \mu \mathrm{g} / \mathrm{ml}$ of TAMO, c) plasma of the breast cancer patient with $3.32 \mu \mathrm{g} / \mathrm{ml}$ of tamoxifen. 